\title{
1 Reported COVID-19 Incidence in Wisconsin High School Athletes
}

$4 \quad$ Phillip Sasser, MD, MS, ${ }^{1}$ Timothy McGuine, PhD, LAT, ${ }^{2}$ Kristin Haraldsdottir, $\mathrm{PhD},{ }^{2}$ Kevin

5 Biese, MA, LAT, ${ }^{3}$ Leslie Goodavish, PA, ${ }^{2}$ Bethany Stevens, ${ }^{3}$ Andrew M. Watson, MD, $\mathrm{MS}^{2}$

14 Address for Correspondence:

15 Andrew Watson

16 Department of Orthopedics and Rehabilitation, Division of Sports Medicine

171685 Highland Avenue

18 Madison, WI 53705

19 Phone: (608) 263-6477

20 Fax: (608) 263-0503

21 Email: Watson@ortho.wisc.edu 
medRxiv preprint doi: https://doi.org/10.1101/2021.02.18.21251986; this version posted February 20, 2021. The copyright holder for this preprint

(which was not certified by peer review) is the author/funder, who has granted medRxiv a license to display the preprint in perpetuity.

It is made available under a CC-BY-NC-ND 4.0 International license.

\section{Contributorship Statement:}

25 Drs. Sasser, Watson, McGuine and Haraldsdottir, Ms. Goodavish and Mr. Biese conceptualized

26 the study, data collection, data analysis and interpretation, drafted the manuscript, and approved

27 the final manuscript as submitted. Ms. Stevens contributed to the data collection, data analysis

28 and interpretation, reviewed the manuscript and approved the final manuscript as submitted. 


\section{ABSTRACT}

30 Introduction: The purpose of this study was to describe the reported incidence of COVID-19 in

31 Wisconsin high school athletes in September 2020, and to investigate the relationship of

32 COVID-19 incidence with sport and face mask use.

33 Methods: Surveys were sent to athletic directors of all Wisconsin high schools regarding sports

34 during September 2020. The association between reported case rates in athletes in each county

35 and the county general population were evaluated with a weighted linear model. Multivariable

36 negative binomial regression models evaluated the associations between COVID-19 incidence

37 and sport type and face mask use by players, adjusting for the county COVID-19 incidence for

38 each school.

39 Results: 207 schools that had reinitiated sport reported 270 COVID-19 cases among 30,074

40 players, for case and incidence rates of 809 cases per 100,000 players and 32.6 cases per 100,000

41 player-days, respectively. The case rates for athletes in each county were positively correlated

42 with the case rates for the county's general population $(\beta=1.14 \pm 0.20, r=0.60, p<0.001)$. One

43 hundred fifteen (55\%) of cases were attributed to household contact, 85 (41\%) to contact outside

44 sport or school, $5(2.4 \%)$ to school contact, and $1(0.5 \%)$ to sport contact. No difference was

45 identified between team and individual sports (incidence rate ratio (IRR) $=1.03[95 \% \mathrm{CI}=0.49$ -

46 2.2], $\mathrm{p}=0.93$ ) or between non-contact and contact sports (IRR=0.53 [0.23-1.3], $\mathrm{p}=0.14)$, although

47 the difference between outdoor and indoor sports approached statistical significance $(\mathrm{IRR}=0.52$

48 [0.26-1.1], $\mathrm{p}=0.07) .84 \%$ of schools required face masks while playing. For those sports with

$49>50$ participating schools, there were no significant associations between COVID-19 incidence

50 and face mask use in cross country (IRR=0.71 [0.2-2.2], $\mathrm{p}=0.52)$, football (IRR=1.6 [0.6-5.1], 
$51 \mathrm{p}=0.404)$, boys soccer $(\operatorname{IRR}=2.3$ [0.5-17], $\mathrm{p}=0.31)$, or girls volleyball $(\operatorname{IRR}=1.4[0.3-6.6]$,

$52 \mathrm{p}=0.64)$.

53 Conclusions: Incidence of reported COVID-19 among athletes was related to background

54 county incidence and most cases were attributed to household and community contact. Although

55 not statistically significant, reported COVID-19 incidence may be lower in outdoor sports. Face

56 mask use did not have a significant benefit, which may be due to relatively low rates of COVID-

$57 \quad 19$ and the small number of schools that did not report using face masks.

58

59 Keywords: adolescent; infection; pediatric; SARS-CoV-2 


\section{INTRODUCTION}

The COVID-19 pandemic has caused unprecedented changes to the daily lives of people

62 of all ages globally. Sports throughout the country have been shut down or altered in varying

63 ways depending on the local county or state ordinances. In Wisconsin, youth sports were

64 effectively canceled by the Safer At Home order in March 2020. ${ }^{1}$ With the order overturned by

65 the Wisconsin Supreme Court in May 2020, counties throughout the state instituted their own

66 restrictions. ${ }^{2,3}$ Youth sports were restarted in certain counties around the state during the summer

67 of 2020, while others did not reinitiate. ${ }^{4}$

68 There has been a dearth of information regarding the risk of COVID-19 infection and

69 sport participation. However, it is widely accepted that transmission of the SARS-CoV-2 virus

70 that causes COVID-19 is related to direct exposure to respiratory droplets and airborne

71 transmission. ${ }^{5,6}$ Longer periods of time in close proximity to infected individuals may increase

72 transmission risk, and high intensity exercise may potentiate the spread of respiratory droplets,

73 according to the United States Center for Disease Control and Prevention (CDC). ${ }^{5}$ National,

74 state, and local regulations have all suggested an associated higher transmission risk with contact

75 sports, indoor sports and team sport participation. ${ }^{7}$ Little direct evidence from sports exists to

76 support this. Media reports of COVID-19 transmission within youth sports are a cause for

77 concern, although it is often unclear whether the risk is due to sport participation or gatherings

78 peripheral to or separate from the sports arena. ${ }^{8,9} \mathrm{~A}$ recent study in a soccer club from

79 Washington state found physically distanced youth soccer training to be safe and did not

80 contribute to COVID-19 spread among child and adolescent participants. ${ }^{10}$ Another preprint

81 publication regarding club soccer players nationwide found no difference in reported COVID-19

82 incidence among athletes participating in contact versus non-contact soccer. ${ }^{11}$ Finally, a third 
83 preprint study of data collected from high school athletic directors nationwide found that,

84 although COVID-19 incidence was higher among indoor sports, very few cases of COVID-19

85 were reportedly attributable to sport contact and the overwhelming majority were attributed to

86 household and community contacts. ${ }^{12}$

87 Several recommendations to minimize COVID-19 risk in youth sports have been

88 published since the onset of the pandemic by various academic organizations, public health

89 agencies, and national sport governing bodies. ${ }^{7,13,14,15,16}$ However, because little data is directly

90 available for sport contexts, these recommendations are largely based on inpatient COVID-19

91 data, case studies, and expert opinion. Risk mitigation recommendations vary widely and the

92 debate around facemask use while playing sports continues. ${ }^{17}$ The CDC and American Academy

93 of Pediatrics (AAP) recommend against the use of facemasks during play if they inhibit

94 breathing, become wet, or become a choking hazard, but strongly recommend the use of

95 facemasks any time while not in the act of playing a sport. ${ }^{14,18}$ Therefore, the purpose of this

96 study is to describe the incidence of reported COVID-19 in high school sports in Wisconsin and

97 to understand the associations between COVID-19 incidence and sport type as well as face mask

98 use among athletes.

100 METHODS

\section{Study Design}

102 All procedures performed in this study were deemed exempt from by the Institutional

103 Review Board of the University of Wisconsin-Madison. In collaboration with the Wisconsin

104 Interscholastic Athletics Association (WIAA), surveys were distributed to all high school athletic

105 directors on October 1, 2020. In addition to school name and location, athletic directors were 
106 asked whether they had restarted participation in sports since the initial COVID-19 restrictions in

107 the spring of 2020. Those schools that reported reinitiating sports were asked to provide the

108 specific sports and the date of restarting, number of athletes, number of practices and games, and

109 number of COVID-19 cases among athletes within each sport, as well as the reported sources of

110 infections (if known) during the month of September 2020. Schools were asked about their type

111 of instruction during September (virtual or in-person) and whether they required the use of face

112 masks for players while playing. Schools were included if they had any sport that had restarted

113 participation during September 2020.

\section{Statistical Analysis}

115 Data were initially evaluated using descriptive statistics, including estimates of central

116 tendency (mean, median) and variability (standard deviation, interquartile range, range) for

117 continuous variables, and counts and percentages for categorical variables. Reported COVID-19

118 case rates were expressed as the number of reported cases per 100,000 players (cases / total

119 number of players * 100,000) overall and for each sport. Duration of participation for each sport

120 at each school was determined as the difference in days between the date of restarting and

121 October 1, 2020, and player-days was determined as the product of the number of participating

122 players and duration. Reported COVID-19 incidence rates were expressed as the number of

123 reported cases per 100,000 player-days (cases / total number of player-days * 100,000) overall

124 and for each sport, with confidence intervals calculated using an exact method.

125 In addition, the number of cases, total population, case rate and incidence rate during

126 September were determined for each county in which a respondent high school was located from

127 publicly available online information from the local health authority. In order to determine

128 whether background county COVID-19 case rates were associated with reported COVID-19 case 
129 rates among high school athletes, the total number of athletes and reported COVID-19 cases

130 were aggregated by county. For those counties with $>100$ athletes, the relationship between

131 COVID-19 case rates among high school athletes and the general population were evaluated with

132 a linear model weighted for the total population of each county.

133 For those sports with data from 50 or more schools, the relative risk of each sport was

134 evaluated using a mixed effects negative binomial regression model to predict the number of

135 COVID-19 cases for each team with local incidence, instructional delivery type, and sport as

136 fixed effects, the log of player-days as an offset, and school as a random effect, yielding an

137 incidence rate ratio (IRR) with "Cheer / Dance" as the reference (since this represented the

138 median unadjusted incidence rate). To evaluate the relationship between reported COVID-19

139 incidence and sport characteristics, a multivariable negative binomial regression model was

140 developed to predict the number of cases, with local incidence, sport location (indoor, outdoor),

141 sport contact (contact, non-contact), sport type (team, individual), and school instructional

142 delivery type as covariates, and the log of player-days as an offset.

143 To evaluate the association between overall COVID-19 incidence and reported face mask

144 use, incidence rates and $95 \%$ confidence intervals were calculated within each sport with greater

145 than 50 reporting schools for those reporting face mask use or not. Separate multivariable

146 negative binomial regression models were then developed to predict the number of cases, with

147 local incidence, instructional delivery type, and face mask use (yes/no) as covariates, and the log

148 of player-days as an offset. Coefficients from the models were exponentiated to represent IRRs

149 for binary variables and Wald confidence intervals were generated. Significance level was

150 determined a priori at the 0.05 level and all tests were 2-tailed. All statistical analyses were

151 performed in $\mathrm{R}$. 


\section{RESULTS}

154 Two hundred forty-four schools submitted complete survey responses, of which 207 had

155 restarted a fall sport. These schools represented 30,074 student-athletes that had participated in

15616,898 practices and 4,378 games. One hundred eighty-seven schools $(90.3 \%)$ reported utilizing

157 in-person instruction during September 2020. Among the schools that had restarted participation,

158270 cases of COVID-19 were reported, yielding a case rate of 892 cases per 100,000 athletes and

159 an incidence rate of $32.6(95 \% \mathrm{CI}=28.9-36.8)$ cases per 100,000 player-days. From September 6

160 to October 4, 2020, 2390 cases of COVID-19 were reported among 14-17 year-olds in

161 Wisconsin, for a case rate of 1067 cases per 100,000 people and an incidence of 38.1 cases per

162100,000 person-days. ${ }^{19}$ Of the cases with a reported known source, $115(55 \%)$ were attributed to

163 household contact followed by community contact outside sport or school $(85,41 \%)$, school

164 contact $(5,2.4 \%)$, sport contact $(1,0.5 \%)$ and other $(3,1.4 \%)$. For those sports with greater than

16550 participating schools, the incidence rate ranged from 13.3 (Tennis - Girls) to 45.2 cases per

166 100,000 player-days (Football), as shown in Figure 1 (full data available in Supplemental Table

167 1).

168 When aggregated by county, the case rates for athletes in each county were significantly

169 and positively correlated with the case rates for their respective county's general population

$170(\beta=1.14 \pm 0.20, r=0.60, p<0.001$; see Figure 2$)$. The IRRs for specific sports, adjusted for state

171 COVID-19 incidence, instruction delivery type and school repeated measures are shown in

172 Figure 3 . The IRRs for school instructional delivery and sport characteristics are shown in Table

173 1. One hundred seventy-three schools (84\%) reported face mask use by players while playing

174 sports. Unadjusted incidence for teams with and without reported face mask use within each 
175 sport with greater than 50 respondent schools are shown in Figure 4. After adjusting for local

176 county COVID-19 incidence and school instructional delivery, face mask use was not associated

177 with a decreased COVID-19 incidence in football, girls' volleyball, boys' soccer or cross country

178 (Table 2).

180 DISCUSSION

181 In this statewide survey study of high school athletes, we did not identify a statistically

182 significant association between COVID-19 incidence and sport type after adjusting for local

183 virus incidence and school instructional delivery. Girls' tennis, girls' golf and cross country

184 reported the lowest adjusted incidence rates of COVID-19, while football reported the highest.

185 Nonetheless, the confidence intervals around these estimates were wide, perhaps due to the

186 relatively low incidence of COVID-19 in Wisconsin during this period. There were no

187 independent, statistically significant differences in reported COVID-19 incidence between indoor

188 versus outdoor sports, contact versus non-contact sports, or individual versus team sports.

189 However, indoor and contact sport classification approached significance, suggesting that this

190 relationship may become clearer with a larger sample during a period of higher COVID-19

191 incidence. In fact, a recent preprint publication among high school athletes nationwide

192 demonstrated this relationship over a longer time period when background COVID-19 rates were

193 higher throughout the country. ${ }^{12}$

194 We found that case rates of reported COVID-19 in high school athletes were significantly

195 related to the case rates of their respective county's general population, and very similar to the

196 overall COVID-19 incidence rate among 14-17 year olds in Wisconsin during roughly the same

197 time frame. In addition, 96\% of the cases among athletes had a reported source of infection from 
198 household and community contacts, and only $1(0.5 \%)$ from a known sport contact. This may

199 suggest that the background, local COVID-19 incidence may have a greater effect on overall

200 COVID-19 incidence among high school athletes than participation in a specific sport or type of

201 sport.

Reported face mask use among the sports with the largest number of respondent schools

203 (football, girls' volleyball, boys' soccer, and cross country) did not have a significant

204 relationship with COVID-19 incidence. While this is similar to the findings among outdoor

205 sports in a recent nationwide sample of high school athletes, it is in contrast to the finding from

206 that study that facemask use was associated with decreased COVID-19 incidence among indoor

207 sports. $^{12}$ The overwhelming majority of the respondent schools in the present study (84\%)

208 reported facemask use while on the field or court. Together with the relatively low background

209 COVID-19 incidence rates during September, this may have limited our ability to identify a true

210 relationship between mask use and reported COVID-19 incidence.

211 Similarly, we did not find a relationship between COVID-19 incidence and type of

212 instructional delivery (in-person, virtual). Of the cases with a reported known source, only $2.5 \%$

213 were attributed to a school contact. This is consistent with prior reports that schools have not

214 been significant contributors to the spread of COVID-19. ${ }^{20}$ However, $90 \%$ of our respondent

215 schools reported in-person instruction, making it difficult to fully evaluate the role of in-person

216 school instruction in COVID-19 incidence among high school athletes. Nonetheless, we included

217 school instruction type within our adjusted models in order to account for this as a potential

218 confounder. Importantly, it should be recognized that this study cannot account for transmission

219 or incidence of COVID-19 among attendees at high school sporting events beyond the

220 participants. This represents an important potential contributor to community COVID-19 risk, 
221 and risk mitigation procedures should continue to be prioritized to protect both athletes and

222 attendees.

\section{Limitations}

225 This study has several limitations. We are unable to verify the information provided by

226 athletic directors through a separate independent source. Local, county-level daily COVID-19

227 case data was often not available for adolescents or children, so our adjusted models could only

228 account for the population-level background incidence from each county. Nonetheless, we found

229 that reported case rates from our sample and the case rates from the county general populations

230 were highly related. It is difficult to fully interpret comparisons of COVID-19 cases reported by

231 athletic directors and those collected by public health agencies, but we have included public

232 health data to add context for our findings and to adjust our incidence models. As mentioned

233 above, the incidence of COVID-19 was relatively low during September 2020 in Wisconsin, and

234 this may have limited our ability to detect statistically significant associations in some cases.

235 Reported sources of infection were provided by the schools themselves and it is unknown

236 whether these represent the results of formal contact tracing by local health authorities. Finally,

237 this data represents information regarding athletes from a single state and may not be

238 generalizable to other populations.

240 Conclusions

241 After adjusting for local county COVID-19 incidence, no statistically significant

242 differences in reported COVID-19 incidence were identified between sports or sport types

243 among Wisconsin high school athletes during September 2020. Reported COVID-19 case rates 
244 among athletes were highly correlated with case rates for the general population for their

245 respective counties, and incidence rates among athletes were very similar to those of 14-17 year

246 olds in Wisconsin in general. Most cases among athletes were attributed to household and

247 community contact with very few attributed to school or sport contacts. Further research is

248 warranted to better define the risk factors for COVID-19 transmission during adolescent sport

249 participation and the relative benefits of different risk mitigation strategies.

\section{ACKNOWLEDGEMENTS}

252 There are no funding sources to report for this study. Dr. Watson serves as the Chief

253 Medical Advisor for the Elite Clubs National League and is supported by grants from the

254 National Center for Advancing Translational Sciences (UL1TR002373; KL2TR002374). We are

255 grateful for the resources and support of the UW Institute for Clinical and Translational

256 Research. Dr. McGuine serves on the Sports Medicine Advisory Council for the National

257 Federation of State High School Associations. There are no other relevant conflicts of interest to 258 disclose. 


\section{REFERENCES}

260 1. COVID-19: Badger Bounce Back. Wisconsin Department of Health Services. Published March 21, 2020. Accessed January 26, 2021. https://www.dhs.wisconsin.gov/covid19/prepare.html

2. Molly Beck, Patrick Marley. Wisconsin Supreme Court rules to strike down Evers' stay-athome order. Milwaukee Journal Sentinel. Published May 13, 2020. Accessed February 2, 2021. https://www.jsonline.com/story/news/politics/2020/05/13/wisconsin-supreme-courtstrikes-down-tony-evers-coronavirus-orders/5179205002/

3. Viviani N, Danbeck J. Dane Co. issues a "Safer at Home"-style order after state one was struck down. https://www.nbc15.com. Accessed February 2, 2021. https://www.nbc15.com/content/news/With-Safer-at-Home-struck-down-Dane-Countyissues-its-own-similar-order-570452141.html

4. Heinrich J. Current Order | Public Health Madison \& Dane County, Public Health Madison \& Dane County. Madison \& Dane County Public Health. Accessed January 26, 2021. /coronavirus/forward-dane/current-order

5. CDC. Coronavirus Disease 2019 (COVID-19) - Transmission. Centers for Disease Control and Prevention. Published October 28, 2020. Accessed January 26, 2021. https://www.cdc.gov/coronavirus/2019-ncov/prevent-getting-sick/how-covid-spreads.html

6. Furukawa NW, Brooks JT, Sobel J. Evidence Supporting Transmission of Severe Acute Infect Dis. 2020;26(7). doi:10.3201/eid2607.201595

7. CDC. COVID-19 and Your Health. Centers for Disease Control and Prevention. Published February 11, 2020. Accessed January 26, 2021. https://www.cdc.gov/coronavirus/2019ncov/daily-life-coping/playing-sports.html

8. Sweeney E. Thirteen COVID-19 cases connected to youth ice hockey camp in New Hampshire - The Boston Globe. BostonGlobe.com. Published September 2, 2020. Accessed January 26, 2021. https://www.bostonglobe.com/2020/09/02/metro/thirteen-covid-19-casesconnected-youth-ice-hockey-camp-new-hampshire/

9. Lazar K. Mounting clusters in youth sports, pandemic fatigue complicate fight against coronavirus in Mass. - The Boston Globe. BostonGlobe.com. Published November 4, 2020. Accessed January 26, 2021. https://www.bostonglobe.com/2020/11/04/metro/mountingclusters-youth-sports-pandemic-fatigue-complicate-fight-against-coronavirus-mass/

10. Drezner JA, Drezner SM, Magner KN, Ayala JT. COVID-19 Surveillance in Youth Soccer During Small Group Training: A Safe Return to Sports Activity. Sports Health. 2021;13(1):15-17. doi:10.1177/1941738120964458 
11. Watson A, Haraldsdottir K, Biese K, Goodavish L, Stevens B, McGuine T. COVID-19 in Youth Soccer. medRxiv. Published online January 1, 2020:2020.09.25.20201616. doi:10.1101/2020.09.25.20201616

12. Watson AM, Haraldsdottir K, Biese K, Goodavish L, Stevens B, McGuine T. The Association of COVID-19 Incidence with Sport and Face Mask Use in United States High School Athletes. medRxiv. Published online January 20, 2021:2021.01.19.21250116. doi:10.1101/2021.01.19.21250116

13. Asif IM, Chang CJ, Diamond AB, Raukar N, Zaremski JL. Returning Athletes Back to High School Sports in the COVID-19 Era: Preparing for the Fall. Sports Health. 2020;12(6):518-520. doi:10.1177/1941738120953851

14. COVID-19 Interim Guidance: Return to Sports. American Academy of Pediatrics. Accessed January 26, 2021. http://services.aap.org/en/pages/2019-novel-coronavirus-covid19-infections/clinical-guidance/covid-19-interim-guidance-return-to-sports/

15. Recommendations for Returning to Play in a Training Environment. Elite Clubs National League. Published May 26, 2020. Accessed January 26, 2021. https://www.theecnl.com/wpcontent/uploads/2020/09/ECNL_x_Return_to_Play_Recs_V5.pdf

16. COVID-19 Return-to-Sport Considerations for Secondary School Athletic Trainers. National Athletic Trainer Association. Published May 2020. Accessed January 26, 2021. https://www.nata.org/sites/default/files/covid_19_return-tosport_considerations_for_secondary_school_ats_1.pdf

17. Karissa Neihoff. Mitigation Efforts Must Intensify as Winter Sports, Performing Arts Begin. National Federation of State High School Associations. Published January 27, 2021. Accessed February 2, 2021. https://www.nfhs.org/articles/mitigation-efforts-must-intensifyas-winter-sports-performing-arts-begin/

18. CDC. Considerations for Wearing Masks. Centers for Disease Control and Prevention. Published December 18, 2020. Accessed January 26, 2021. https://www.cdc.gov/coronavirus/2019-ncov/prevent-getting-sick/cloth-face-coverguidance.html

19. COVID-19: Wisconsin Cases. Wisconsin Department of Health Services. Published February 17, 2021. Accessed February 17, 2021. https://www.dhs.wisconsin.gov/covid19/cases.htm

20. Falk A, Benda A, Falk P, Steffen S, Wallace Z, Høeg TB. COVID-19 Cases and Transmission in $17 \mathrm{~K}-12$ Schools - Wood County, Wisconsin, August 31-November 29, 2020. MMWR Morb Mortal Wkly Rep. 2021;70(4):136-140. doi:10.15585/mmwr.mm7004e3 
331 Figure Legends.

333 Figure 1. Unadjusted incidence rates of COVID-19 during September 2020 for various sports

334 from Wisconsin high schools. Incidence rate is shown as reported cases per 100,000 player-days

335 for those sports with greater than 50 schools reporting restarting. Red, dashed line represents the

336 COVID-19 incidence rate among 14-17 year-olds in Wisconsin from 9/6 to 10/5/2020 (data

337 extracted from https://www.dhs.wisconsin.gov/covid-19/cases.htm\#youth on 2/5/2021).

338 Figure 2. Reported COVID-19 case rates for Wisconsin high school athletes and the general

339 population of their respective counties during September 2020. Size of points scaled to

340 population of each county and dashed line represents a line of equality. Solid line and shaded

341 area represent regression line and 95\% confidence interval from linear model weighted for

342 population of each county. $r=$ correlation coefficient.

343 Figure 3. COVID-19 incidence rate ratios during September 2020 for Wisconsin high school

344 sports, adjusted for local (state) COVID-19 incidence, instructional delivery type and repeated

345 measures from the same school. Includes those sports with greater than 50 schools reporting

346 participation, with Cheer/Dance as reference. ${ }^{*} \mathrm{p}<0.05$.

347 Figure 4. Unadjusted COVID-19 incidence rates reported among Wisconsin athletes in

348 September 2020, comparing teams with or without reported face mask use, within each sport.

349 Includes those sports with greater than 50 reporting schools. 
351 Table 1. Incidence rate ratios for reported COVID-19 cases among Wisconsin high schools in

352 fall 2020 by school instructional delivery and sport characteristics.

\begin{tabular}{lcc}
\hline & IRR $(95 \%$ CI $)$ & $\mathrm{p}$ \\
\hline School instructional delivery (in-person) & $1.33(0.74-2.4)$ & 0.34 \\
Outdoor & $0.52(0.26-1.1)$ & 0.072 \\
Team & $1.03(0.49-2.2)$ & 0.93 \\
Non-Contact & $0.53(0.23-1.26)$ & 0.14
\end{tabular}

$353{ }^{a}$ Incidence rate ratios and Wald confidence intervals from a mixed effects negative binomial

354 regression to predict COVID-19 cases with local incidence, instructional delivery type, and sport

355 characteristics as fixed effects, school as a random effect and $\log$ (player-days) as an offset; $\mathrm{CI}=$

356 Confidence Interval; IRR=Incidence Rate Ratio. 
358 Table 2. The association of reported face mask use with COVID-19 incidence within each sport

359 among Wisconsin high school athletes during September 2020. ${ }^{\mathrm{a}}$

\begin{tabular}{lcc}
\hline & IRR $(95 \% \text { CI })^{\mathrm{a}}$ & $\mathrm{p}$ \\
\hline Cross Country & $0.706(0.24-2.2)$ & 0.524 \\
Football & $1.58(0.57-5.1)$ & 0.404 \\
Soccer - Boys & $2.34(0.52-17)$ & 0.307 \\
Volleyball - Girls & $1.37(0.3-6.6)$ & 0.641 \\
\hline
\end{tabular}

$360{ }^{\mathrm{a}}$ Incidence rate ratios and Wald confidence intervals from separate multivariable negative

361 binomial regression models within each sport to predict COVID-19 cases with local incidence,

362 instructional delivery type, and face mask use (yes/no) as fixed effects, and log(player-days) as

363 an offset. $\mathrm{CI}=$ Confidence Interval; IRR=Incidence Rate Ratio. 


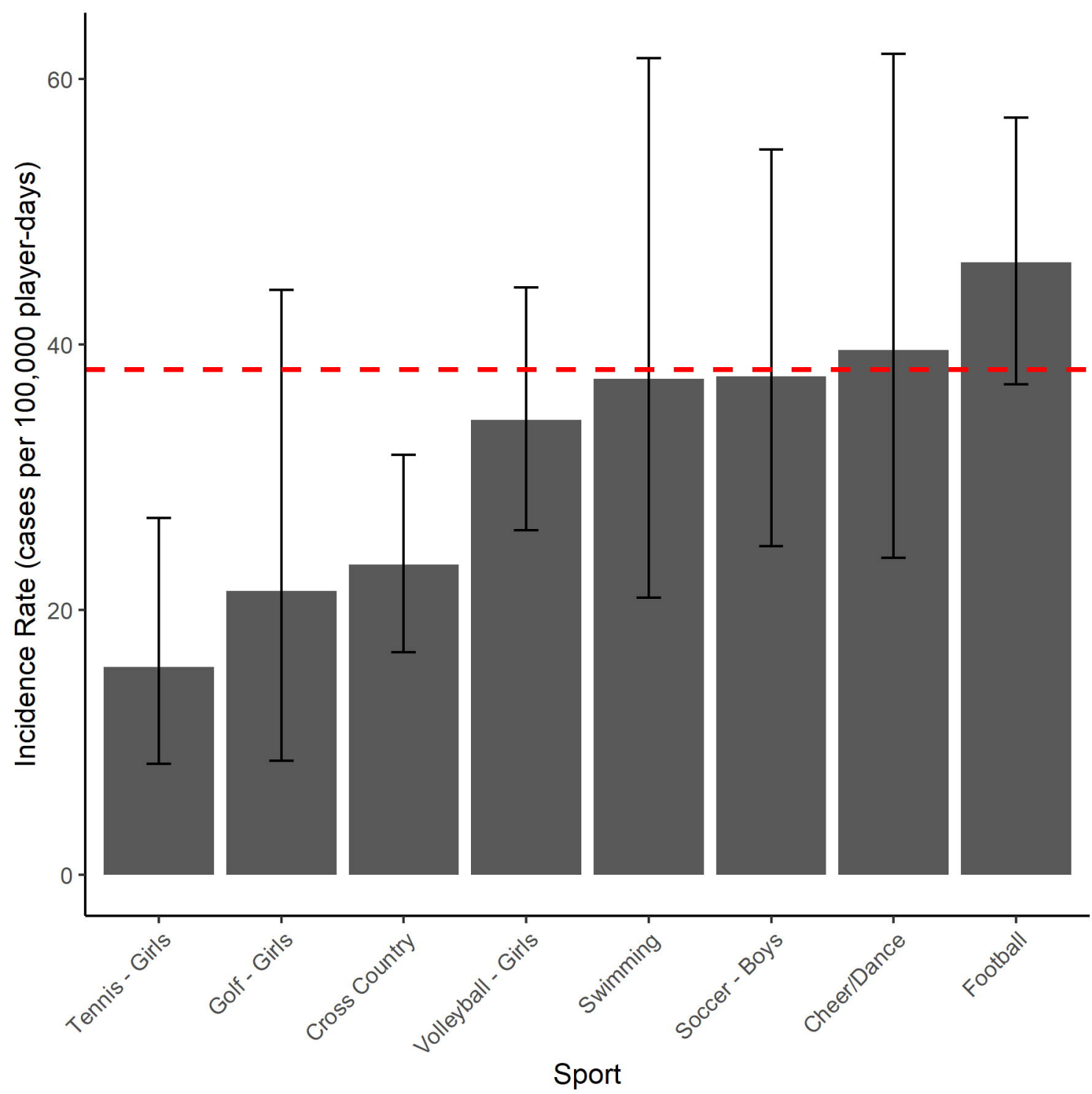




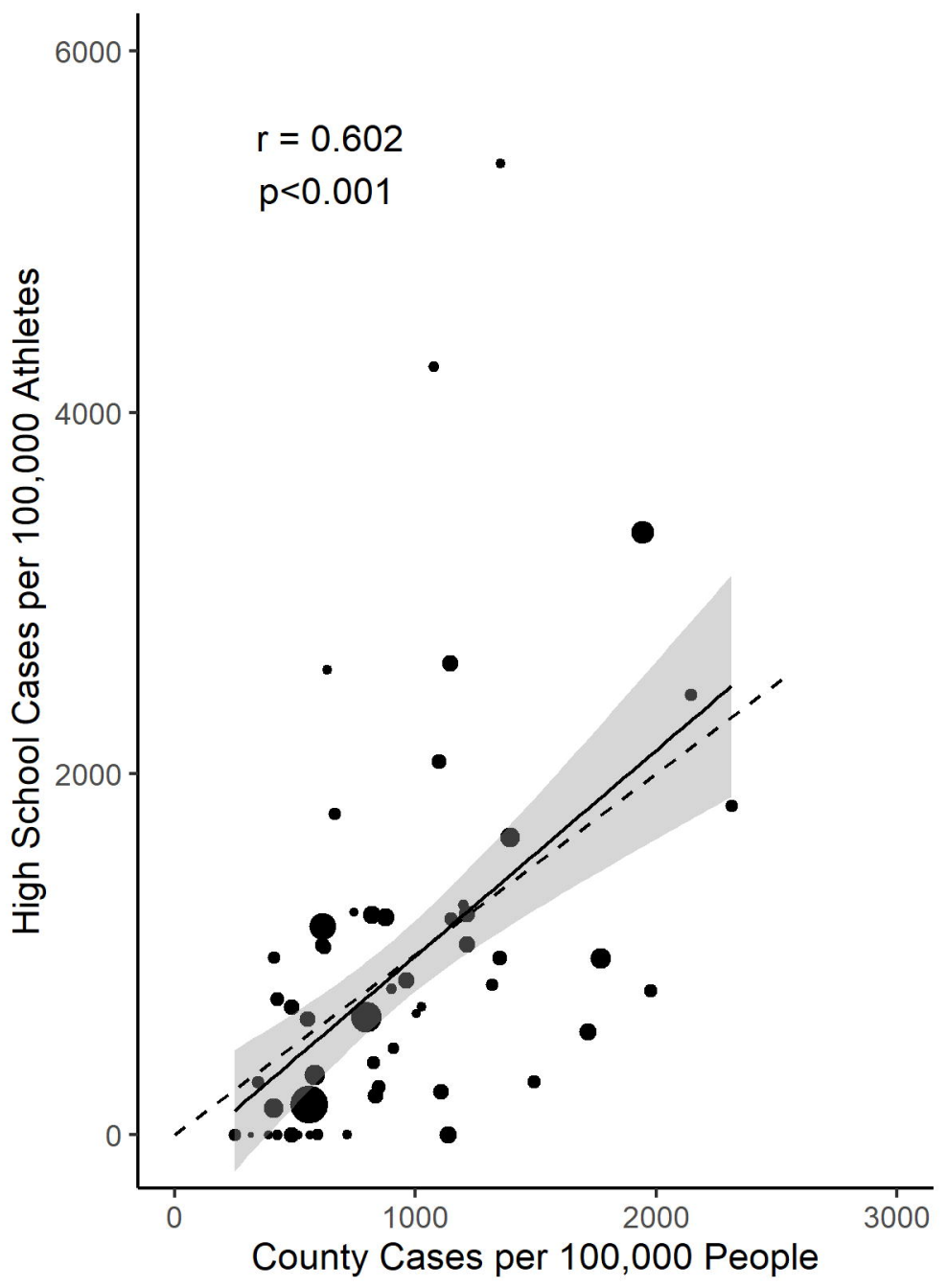




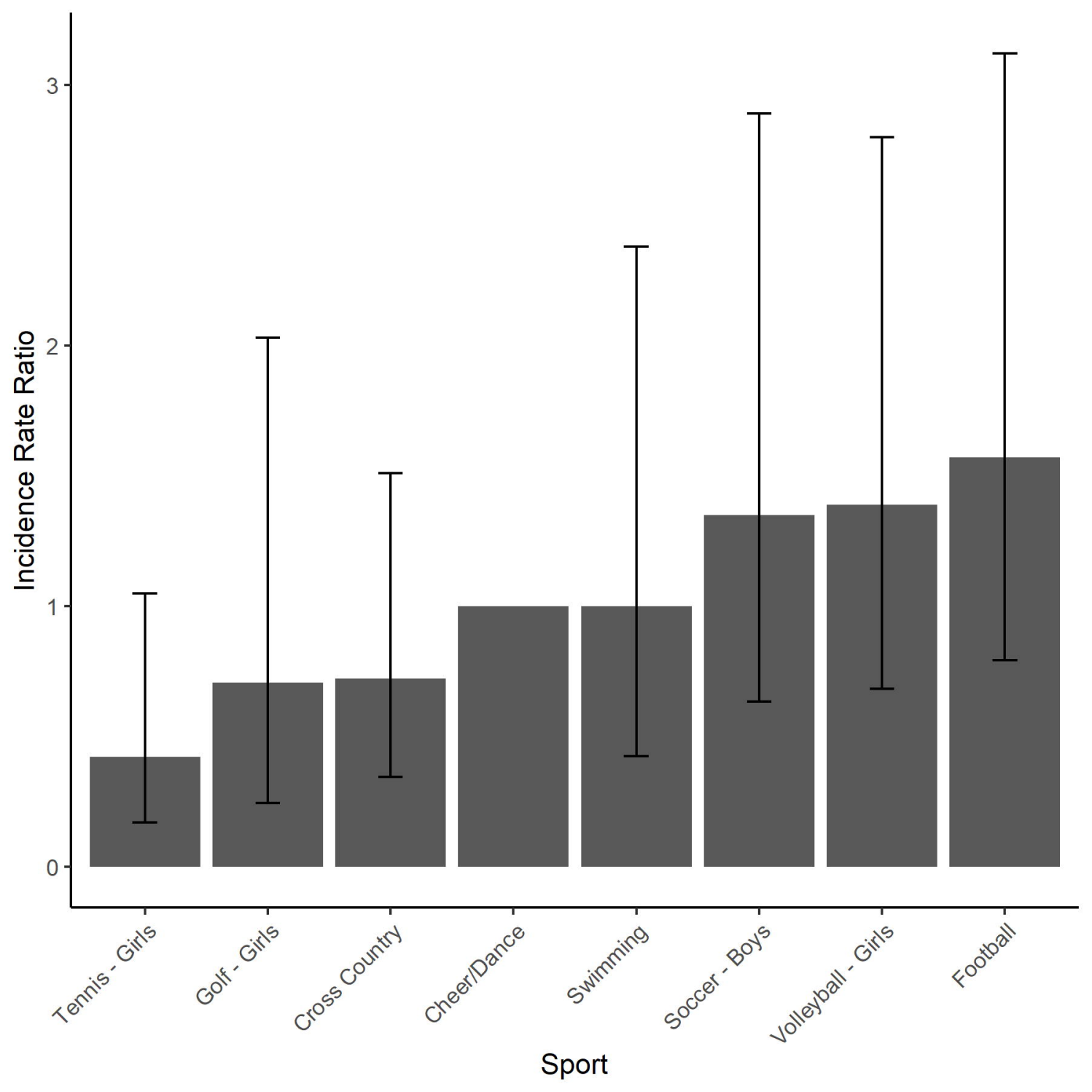


